\title{
Population Dynamic Study of Prey-Predator Interactions with Weak Allee Effect, Fear Effect, and Delay
}

\author{
Ye Xuan Li $\mathbb{D}^{1},{ }^{1}$ Hua Liu $\left(\mathbb{D},{ }^{1}\right.$ Yu Mei Wei $\mathbb{D}^{2},{ }^{2}$ Ming Ma $\mathbb{D}^{1},{ }^{1}$ Gang Ma, ${ }^{1}$ and Jing Yan Ma ${ }^{3}$ \\ ${ }^{1}$ School of Mathematics and Computer Science, Northwest Minzu University, Lanzhou 730000, China \\ ${ }^{2}$ Experimental Teaching Department, Northwest Minzu University, Lanzhou 730030, China \\ ${ }^{3}$ School of Preparatory Education, Northwest Minzu University, Lanzhou 730030, China \\ Correspondence should be addressed to Hua Liu; 7783360@qq.com
}

Received 13 July 2021; Revised 27 September 2021; Accepted 6 January 2022; Published 9 February 2022

Academic Editor: Ljubisa Kocinac

Copyright (c) 2022 Ye Xuan Li et al. This is an open access article distributed under the Creative Commons Attribution License, which permits unrestricted use, distribution, and reproduction in any medium, provided the original work is properly cited.

In this study, a predator-prey model with the Allee effect and fear effect is established. We use the comparison principle to prove boundedness. The zero equilibrium point and nonzero equilibrium point of the model are calculated, and the local stability conditions are obtained. Next, according to the Sotomayor theorem, the cross-sectional conditions of transcritical bifurcation and Hopf bifurcation are obtained. The conditions for the Hopf bifurcation to be supercritical or subcritical can be calculated by the normal form theory. Then, to make the model more realistic, we introduce the gestation delay in the proposed mathematical model. Stability and Hopf bifurcation are also analyzed. Finally, several numerical simulations are presented to verify the conclusions. Our results demonstrate that the Allee effect, fear effect, and delay play significant roles in population dynamics. The Allee effect and delay destabilize the originally stable model, after which Hopf bifurcation occurs. However, the fear effect can enhance stable coexistence.

\section{Introduction}

In mathematical ecology, the interaction between the predator and prey is important and complex and will continue to be predominant in ecology because it is widespread globally. Since the establishment of the well-known Lotka-Volterra model solved the key problems of ecological processes [1, 2], applied mathematicians usually use mathematical models as tools to study the complex dynamics between predators and prey. It is an effective method to study the dynamic behavior of the population by establishing realistic mathematical models. Different types of models are proposed to investigate the interactions in mathematical biology. The classic predator-prey model can be expressed by the following first-order ordinary differential equations:

$$
\begin{aligned}
& \frac{\mathrm{d} x}{\mathrm{~d} t}=x l(x)-g(x, y) y, \\
& \frac{\mathrm{d} y}{\mathrm{~d} t}=-\delta y+h(x, y) y,
\end{aligned}
$$

where $x$ and $y$ are the population of prey and predator, respectively, $l(x)$ is the birth rate of prey, and $\delta$ is the predator death rate. Here, the role of the functional response term is to connect the predator and the prey, that is, the prey biomass consumed by each predator in unit time, which is described by $g(x, y)$, while $h(x, y)$ is called the numerical response of the predator [3], which means the growth rate of the predator realized by consuming the prey. Holling proposed several different functional response types. Because this method can reflect the interspecies relationship between the predator and prey in the model, it has wide applicability. Many scholars incorporate this method into mathematical ecology modeling. Holling type-I, type-II, and type-III functional responses have been extensively studied [3-5]. Usually, $g(x, y)$ is called a prey-dependent functional response, which is a function that considers the density of the prey. We choose a simple linear function $g(x)=a x$, that is, the Holling type-I functional response in this paper, where $a$ is the predator predation rate. In the traditional system, $g(\cdot)=h(\cdot)$; however, in a dissipative system, $h(x, y)$ $=c g(x, y)$, where $c$ is the prey-to-predator conversion rate 
and $0<c<1$. As is well known, restricted by the competition of the same species, environmental factors, and other mechanisms, populations in nature cannot grow indefinitely. Allowing the prey population to grow logically is a common modeling method and has the form $x l(x)=x r(1-(x / K))$, where $r$ is the intrinsic growth rate of prey and $K$ is the carrying capacity. In summary, the interaction between the predator and prey can be expressed by the following equations:

$$
\begin{aligned}
& \frac{\mathrm{d} x}{\mathrm{~d} t}=r x\left(1-\frac{x}{K}\right)-a x y, \\
& \frac{\mathrm{d} y}{\mathrm{~d} t}=\operatorname{cax} y-\delta y .
\end{aligned}
$$

Taking the long-term view, predators can impact the population density of prey only through direct killing. However, the mere presence of predators can change the physiology of prey and even reduce its birth rate. This phenomenon can exert an effect on the population density of prey that is even more obvious than direct predation and is called the fear effect [6-8]. Some animals show a series of antipredator responses, such as habitat migration and foraging behavior change. For example, birds have changed habitats because of a fear of predators in the same habitat. Although new habitats are safer, the relocated animals are unable to adapt to new habitats, and a decline in population ensues [9, 10]. Wang et al. [11] established a model with a fear effect and Holling functional response. They first formulated the fear-effect term $c$ and combined the fear factor with a predator-prey model widely [12]. Here, $f$ represents the fear level that depends on the predator $y$. The fear effect is introduced to further change the prey birth rate by the following equation:

$$
\frac{\mathrm{d} x}{\mathrm{~d} t}=n(f, y) x l(x)
$$

and $n(f, y)$ must meet the following conditions before it can be used as a fear function [11]:

$$
\left\{\begin{array}{l}
n(0, y)=1, \\
n(f, 0)=1, \\
\lim _{f \longrightarrow \infty} n(f, y)=0 \\
\lim _{y \rightarrow \infty} n(f, y)=0 \\
\frac{\partial n(f, y)}{\partial f}<0 \\
\frac{\partial n(f, y)}{\partial y}<0
\end{array}\right.
$$

A simple function $n(f, y)=(1 / 1+f y)$ satisfies all the above conditions.

In population dynamics, the cluster lifestyle of biological populations is beneficial to the growth of populations, but the excessive density of clusters often causes the growth of biological populations to be suppressed due to competition for resources. The population density is too sparse or too dense to be unfavorable for the survival and development of the population. For each population, there must be an independent optimal density for growth and reproduction; this mechanism is called the Allee effect [13-15]. The Allee effect is generally divided into two types: strong and weak $[16,17]$. When the population has a weak Allee effect, although the population density grows slowly, the increase in population density is positive. However, when a species with a sparse population density has a strong effect, the population will eventually become extinct. Since the Allee effect is widely present in natural populations, taking it into account in modeling will make the model more complete. Considering the weak Allee effect $\phi(x)=(x / x+A)(A>0)[18-20]$ and the fear effect, a further modification is required.

$$
\begin{aligned}
& \frac{\mathrm{d} x}{\mathrm{~d} t}=\frac{r x}{1+f y}\left(1-\frac{x}{K}\right) \frac{x}{x+A}-a x y, \\
& \frac{\mathrm{d} y}{\mathrm{~d} t}=\operatorname{cax} y-\delta y .
\end{aligned}
$$

To intuitively understand the impact of fear on the model, the relation between $\mathrm{d} x / \mathrm{d} t$ and $f$ is shown in Figure 1 [21]. With the increase of the fear parameter, the value of $\mathrm{d} x / \mathrm{d} t$ decreases.

There are many types of time delay in the biological models of predators and prey. It is considered herein that the reproduction of the prey population will not be completed instantly and that there is a gestation delay $\tau$ required for gestation of prey $[22,23]$. To make model (5) closer to reality, gestation delay is introduced as follows:

$$
\begin{aligned}
\frac{\mathrm{d} x(t)}{\mathrm{d} t} & =\frac{r x(t)}{1+f y(t)}\left(1-\frac{x(t-\tau)}{K}\right) \frac{x(t)}{x(t)+A}-a x(t) y(t), \\
\frac{\mathrm{d} y(t)}{\mathrm{d} t} & =\operatorname{cax}(t) y(t)-\delta y(t) .
\end{aligned}
$$

Human beings have a long history of using mathematics to explore the world of biological populations and have made tremendous progress. Nowadays, mathematical models have been widely used in many fields. Among them, there are many works on the application of mathematical models to study the dynamics of populations and infectious diseases [24-26]. Establishing mathematical models is a widely used and effective method of studying the dynamic behavior of populations. The advantage of this method is that it is convenient to use the computer to process the main variables and parameters of a model, save time and cost, and make predictions based on past and present information [27]. The fear effect and Allee effect are ecological effects that actually exist in populations, so incorporating rich ecological 


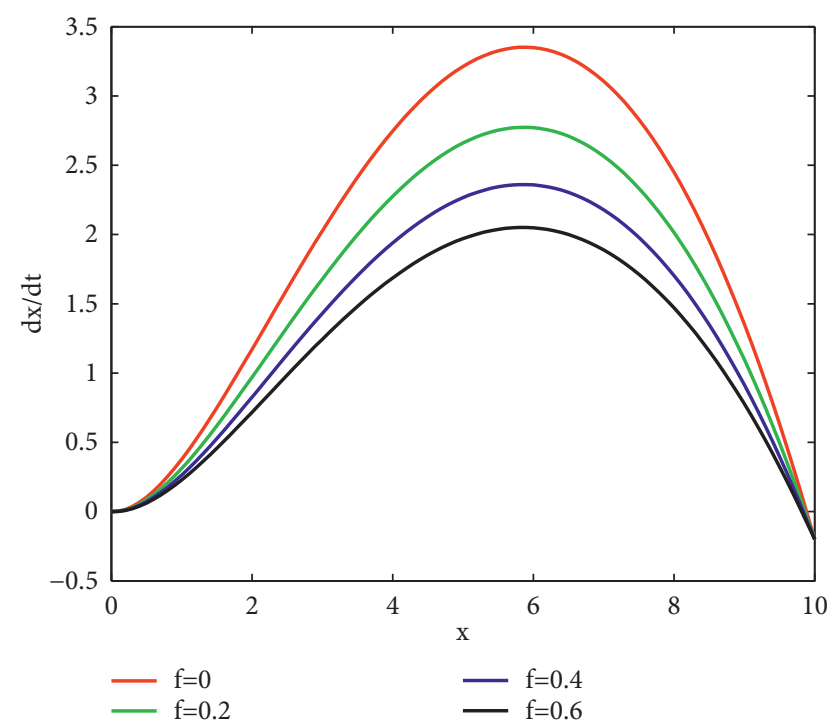

Figure 1: Relation between $\mathrm{d} x / \mathrm{d} t$ and $f$ of model (5) $(y=1$, $r=2.65, K=10, A=5, a=0.02, c=0.22$, and $\delta=1.04$ ).

effects into the mathematical model and constructing a suitable model to study the impact of ecological effects on the dynamic behavior of the model are our research motivation. Although our model is not complicated, an overly complicated model will not be able to facilitate a detailed analysis due to the limitations of research methods. The objective of this study is to explore how these ecological effects will affect the dynamic behavior of the population when there are Allee effects, fear effects, and time delay in the model. In addition, it will be interesting to explore the bifurcation caused by the Allee effect and time delay.

\section{Analysis of the Predator-Prey Model}

Model (5) has three equilibrium points $E_{0}=(0,0)$, $E_{K}=(K, 0)$, and $E_{*}=\left(x_{*}, y_{*}\right)$. Here, $x_{*}=(\delta / a c)$ and $y_{*}=\left(\sqrt{1+4 f r x_{*}\left(K-x_{*}\right) / K a\left(x_{*}+A\right)}-1 / 2 f\right)$. According to the actual meaning of the parameters, $(\delta / a c)<K$ is obtained.

\subsection{Boundedness}

Theorem 1. The solution $(x(t), y(t))$ of the model satisfies

$$
\lim _{t \longrightarrow \infty} \sup \left(x(t)+\frac{1}{c} y(t)\right) \leq \frac{K}{4 r \delta}(\delta+r)^{2} .
$$

Proof. Define $\Lambda=x(t)+(1 / c) y(t)$.

$$
\begin{aligned}
\frac{\mathrm{d} \Lambda}{\mathrm{d} t} & =\frac{\mathrm{d} x}{\mathrm{~d} t}+\frac{1}{c} \frac{\mathrm{d} y}{\mathrm{~d} t} \\
& =\frac{r x}{1+f y}\left(1-\frac{x}{K}\right) \frac{x}{x+A}-a x y+\frac{1}{c} c(a x) y-\frac{1}{c} \delta y \\
& =\frac{r x}{1+f y}\left(1-\frac{x}{K}\right) \frac{x}{x+A}-\frac{1}{c} \delta y .
\end{aligned}
$$

Then, for all $t>0$,

$$
\begin{aligned}
\frac{\mathrm{d} \Lambda}{\mathrm{d} t}+\delta \Lambda & =\frac{r x}{1+f y}\left(1-\frac{x}{K}\right) \frac{x}{x+A}-\frac{1}{c} \delta y+\delta x+\frac{1}{c} \delta y \\
& =\frac{r x}{1+f y}\left(1-\frac{x}{K}\right) \frac{x}{x+A}+\delta x \\
& \leq r x\left(1-\frac{x}{K}\right)+\delta x \\
& =x\left(r+\delta-\frac{r x}{K}\right) \\
& \leq \frac{K}{4 r}(\delta+r)^{2} .
\end{aligned}
$$

Thus,

$$
\lim _{t \rightarrow \infty} \sup \left(x(t)+\frac{1}{c} y(t)\right) \leq \frac{K}{4 r \delta}(\delta+r)^{2} .
$$

\subsection{Stability Analysis}

\section{Theorem 2.}

(1) Trivial equilibrium point $E_{0}$ is stable.

(2) If $a>(\delta / c K)$, the axial equilibrium point $E_{K}$ is a saddle point and is stable, otherwise.

(3) Interior equilibrium $E_{*}$ is locally asymptotically stable for

$$
A<\frac{r x_{*}\left(2 K-3 x_{*}\right)+\sqrt{r^{2} x_{*}^{2}\left(2 K-3 x_{*}\right)^{2}+4 a y_{*} K\left(1+f y_{*}\right) r x_{*}^{2}\left(x_{*}-K\right)}}{2 a y_{*} K\left(1+f y_{*}\right)}-\frac{\delta}{a c},
$$

and $E_{*}$ is unstable, otherwise.

Proof. Let

$$
\begin{aligned}
& F(x, y)=\frac{r x}{1+f y}\left(1-\frac{x}{K}\right) \frac{x}{x+A}-a x y, \\
& G(x, y)=c(a x) y-\delta y .
\end{aligned}
$$

The Jacobian matrix of model (3) is given by

$$
J=\left(\begin{array}{ll}
\frac{\partial F}{\partial x} & \frac{\partial F}{\partial y} \\
\frac{\partial G}{\partial x} & \frac{\partial G}{\partial y}
\end{array}\right),
$$


where

$$
\begin{aligned}
& \frac{\partial F}{\partial x}=\frac{K r x(2 K-3 x)(1+f y)(x+A)+r x^{2} K(x-K)(1+f y)}{[K(1+f y)(x+A)]^{2}}-a y, \\
& \frac{\partial F}{\partial y}=\frac{r x^{2} f(x-K)}{K(x+A)(1+f y)^{2}}-a x, \\
& \frac{\partial G}{\partial x}=c a y, \\
& \frac{\partial G}{\partial y}=c a x-\delta .
\end{aligned}
$$

The community matrix at $E_{0}=(0,0), E_{K}=(K, 0)$, and $E_{*}=\left(x_{*}, y_{*}\right)$ is given by

$$
\begin{aligned}
J_{E_{0}} & =\left(\begin{array}{cc}
0 & 0 \\
0 & -\delta
\end{array}\right), \\
J_{E_{K}} & =\left(\begin{array}{cc}
\frac{-r K}{(1+f y)(K+A)} & -a K \\
0 & c a K-\delta
\end{array}\right), \\
J_{E_{*}} & =\left(\begin{array}{cc}
\frac{K r x_{*}\left(2 K-3 x_{*}\right)\left(x_{*}+A\right)+r x_{*}^{2} K\left(x_{*}-K\right)}{K^{2}\left(1+f y_{*}\right)\left(x_{*}+A\right)^{2}}-a y_{*} & \frac{r x_{*}^{2} f\left(x_{*}-K\right)}{K\left(x_{*}+A\right)\left(1+f y_{*}\right)^{2}}-\frac{\delta}{c} \\
c a y_{*} & 0
\end{array}\right) .
\end{aligned}
$$

It is found that $E_{0}$ is stable. The first eigenvalue of $J_{E_{K}}$ is negative. If $a<(\delta / c K), E_{K}$ is stable, and if $a>(\delta / c K), E_{K}$ is a saddle point.

Next, the community matrix at the interior equilibrium $E_{*}$ is considered. The characteristic polynomial of $J_{E_{*}}$ is

$$
\begin{aligned}
\operatorname{Tr}\left(J_{E_{*}}\right) & =\frac{K r x_{*}\left(2 K-3 x_{*}\right)\left(x_{*}+A\right)+r x_{*}^{2} K\left(x_{*}-K\right)}{K^{2}\left(1+f y_{*}\right)\left(x_{*}+A\right)^{2}}-a y_{*}, \\
\operatorname{Det}\left(J_{E_{*}}\right) & =\delta a y_{*}-\frac{r x_{*}^{2} f\left(x_{*}-K\right) c a y_{*}}{K\left(x_{*}+A\right)\left(1+f y_{*}\right)^{2}} .
\end{aligned}
$$

$$
H(\lambda)=\lambda^{2}-\operatorname{Tr}\left(J_{E_{*}}\right) \lambda+\operatorname{Det}\left(J_{E_{*}}\right),
$$

where

One must obtain the conditions of $\operatorname{Tr}\left(J_{E_{*}}\right)<0 \operatorname{Det}\left(J_{E_{*}}\right)$ $>0$; that is, if

$$
A<\frac{r x_{*}\left(2 K-3 x_{*}\right)+\sqrt{r^{2} x_{*}^{2}\left(2 K-3 x_{*}\right)^{2}+4 a y_{*} K\left(1+f y_{*}\right) r x_{*}^{2}\left(x_{*}-K\right)}}{2 a y_{*} K\left(1+f y_{*}\right)}-\frac{\delta}{a c}
$$

$E_{*}$ is locally asymptotically stable. If

$$
A>\frac{r x_{*}\left(2 K-3 x_{*}\right)+\sqrt{r^{2} x_{*}^{2}\left(2 K-3 x_{*}\right)^{2}+4 a y_{*} K\left(1+f y_{*}\right) r x_{*}^{2}\left(x_{*}-K\right)}}{2 a y_{*} K\left(1+f y_{*}\right)}-\frac{\delta}{a c},
$$

$E_{*}$ is unstable. 


\subsection{Bifurcation Analysis}

\subsubsection{Transcritical Bifurcation}

Theorem 3. At $a=a_{0}$, where $a_{0}=(\delta / c K)$, model (5) enters into transcritical bifurcation around $E_{K}$.
Proof. The eigenvalues of $J_{E_{K}}$ are $(-r K /(1+f y)(K+A))$ and $\lambda_{2}=c a K-\delta . \lambda_{1}$ is negative when $a=(\delta / c K)=a_{0}$ and $\lambda_{2}=0$. Thus, $J_{E_{K}}$ has a zero eigenvalue. $V$ and $W$ are the eigenvectors corresponding to the zero eigenvalue of the matrices $J_{E_{K}}$ and $J_{E_{K}}^{T}$, where $V=((-a(1+f y)(K+A)$ $(r), 1)^{T}$ and $W=(0,1)^{T}$.

$$
\begin{aligned}
J_{E_{K}}^{T} & =\left(\begin{array}{cc}
\frac{-r K}{(1+f y)(K+A)} & 0 \\
-a K & 0
\end{array}\right), \\
W^{T} f_{a}(\bar{x}, \bar{y}, \bar{a}) & =0, \\
W^{T}\left[D f_{a}(\bar{x}, \bar{y}, \bar{a}) V\right] & =c K \neq 0, \\
W^{T}\left[D^{2} f_{a}(\bar{x}, \bar{y}, \bar{a})(V, V)\right] & =\frac{-2 c a^{2}(1+f y)(K+A)}{r} \neq 0 .
\end{aligned}
$$

By the Sotomayor theorem [28], the model attains transcritical bifurcation around $E_{K}$ at $a=a_{0}$.
2.3.2. Hopf Bifurcation. Model (5) undergoes bifurcation when

$$
A=\frac{r x_{*}\left(2 K-3 x_{*}\right)+\sqrt{r^{2} x_{*}^{2}\left(2 K-3 x_{*}\right)^{2}+4 a y_{*} K\left(1+f y_{*}\right) r x_{*}^{2}\left(x_{*}-K\right)}}{2 a y_{*} K\left(1+f y_{*}\right)}-\frac{\delta}{a c}
$$

The model (5) undergoes a Hopf bifurcation will be proved in this section.
The parameter $A_{0}$ is chosen to analyze the Hopf bifurcation at $E_{*}=\left(x_{*}, y_{*}\right)$, where

$$
A_{0}=\frac{r x_{*}\left(2 K-3 x_{*}\right)+\sqrt{r^{2} x_{*}^{2}\left(2 K-3 x_{*}\right)^{2}+4 a y_{*} K\left(1+f y_{*}\right) r x_{*}^{2}\left(x_{*}-K\right)}}{2 a y_{*} K\left(1+f y_{*}\right)}-\frac{\delta}{a c} .
$$

When $A=A_{0}$, one has $T\left(J_{E}\right)=0$, and $\lambda= \pm i$ $\sqrt{\left(2 K f\left(x_{*}+A\right) c a y_{*} /\left[K\left(1+f y_{*}\right)\left(x_{*}+A\right)\right]^{2}\right)+\delta a y_{*}}$ are imaginary eigenvalues of the Jacobian matrix $J_{E_{*}}$. Let $\lambda=$ $\alpha(A) \pm i \beta(A)$ be the roots of $\lambda^{2}-T\left(J_{E_{*}}\right)+D\left(J_{E_{*}}\right)=0$,

$$
\begin{aligned}
\alpha^{2}-\beta^{2}-a T+D & =0 \\
2 \alpha \beta-T \beta & =0
\end{aligned}
$$

Then,

$$
\begin{aligned}
\alpha & =\frac{T}{2} \\
\beta & =\frac{\sqrt{4 D-T^{2}}}{2}, \\
\left.\frac{d \alpha}{d A}\right|_{A=A_{0}} & =\frac{1}{2}\left[\frac{d}{d A} \operatorname{Tr}\left(J_{E_{*}}\right)\right]_{A=A_{0}} \neq 0 .
\end{aligned}
$$

The cross-sectional condition is satisfied, so model (3) undergoes a Hopf bifurcation at $E_{*}=\left(x_{*}, y_{*}\right)$ when $A=A_{0}$. 
Next, the detailed nature of the Hopf bifurcation is analyzed. Transforming the equilibrium point $E_{*}$ into the origin by setting $x=X+x_{*}$ and $y=Y+y_{*}$, one has the following model:

$$
\begin{aligned}
\frac{\mathrm{d} X}{\mathrm{~d} t}= & \frac{r\left(X+x_{*}\right)}{1+f\left(Y+y_{*}\right)}\left(1-\frac{\left(X+x_{*}\right)}{k}\right) \frac{\left(X+x_{*}\right)}{\left(X+x_{*}\right)+A} \\
& -a\left(X+x_{*}\right)\left(Y+y_{*}\right), \\
\frac{\mathrm{d} Y}{\mathrm{~d} t}= & c a\left(X+x_{*}\right)\left(Y+y_{*}\right)-\delta\left(Y+y_{*}\right) .
\end{aligned}
$$

Expanding Taylor's series of the above model at $(X, Y)=(0,0)$

$$
\begin{aligned}
\dot{X}= & c_{10} X+c_{01} Y+c_{20} X^{2}+c_{11} X Y+c_{02} Y^{2}+c_{30} X^{3} \\
& +c_{21} X^{2} Y+c_{12} X Y^{2}+c_{03} Y^{3}+o \\
\dot{Y}= & d_{10} X+d_{01} Y+d_{20} X^{2}+d_{11} X Y+d_{02} Y^{2} \\
& +d_{30} X^{3}+d_{21} X^{2} Y+d_{12} X Y^{2}+d_{03} Y^{3}+o
\end{aligned}
$$

$$
\begin{aligned}
& c_{10}=\frac{K r x_{*}\left(2 K-3 x_{*}\right)\left(1+f y_{*}\right)\left(x_{*}+A\right)+r x_{*}^{2} K\left(x_{*}-K\right)\left(1+f y_{*}\right)}{\left[K\left(1+f y_{*}\right)\left(x_{*}+A\right)\right]^{2}}-a y_{*}, \\
& c_{01}=\frac{r x_{*}^{2} f\left(x_{*}-K\right)}{K\left(x_{*}+A\right)\left(1+f y_{*}\right)^{2}}-\frac{\delta}{c} \\
& c_{20}=\frac{r\left(2 K-6 x_{*}\right)\left(1+f y_{*}\right)^{2}\left(x_{*}+A\right)^{2}-r x_{*}\left(2 K-3 x_{*}\right)\left(1+f y_{*}\right)^{2}\left(x_{*}+A\right)-2 r x_{*}^{2}\left(x_{*}-K\right)\left(1+f y_{*}\right)^{2}}{2 K\left[\left(1+f y_{*}\right)\left(x_{*}+A\right)\right]^{3}}, \\
& c_{11}=-\frac{f r x_{*}\left(2 K-3 x_{*}\right)\left(x_{*}+A\right)+f r x_{*}^{2}\left(x_{*}-1\right)}{2 K\left[\left(1+f y_{*}\right)\left(x_{*}+A\right)\right]^{2}}-a, \\
& c_{02}=\frac{-2 r x_{*}^{2} f^{2}\left(x_{*}-K\right)}{K\left(x_{*}+A\right)\left(1+f y_{*}\right)^{3}}, \\
& c_{30}=\frac{r\left(15 x_{*}+6 A-2 K\right)\left(x_{*}+A\right)^{2}+2 r x_{*}\left(x_{*}-2 A\right)\left(x_{*}-K\right)-r x_{*}\left(2 K-3 x_{*}\right)}{12 K\left(x_{*}+A\right)^{4}\left(1+f y_{*}\right)}+\frac{r\left(2 x_{*}+A\right)\left(2 K-3 x_{*}\right)-2 r x_{*}^{2}}{12 K\left(x_{*}+A\right)^{3}\left(1+f y_{*}\right)}, \\
& c_{21}=\frac{-f r\left(2 K-6 x_{*}\right)\left(x_{*}+A\right)^{2}\left(1+f y_{*}\right)+2 f r x_{*}^{2}\left(x_{*}-K\right)\left(1+f y_{*}\right)}{2 K\left[\left(1+f y_{*}\right)\left(x_{*}+A\right)\right]^{3}}+\frac{+f r\left(3 x_{*}-2\right)\left(2 K-3 x_{*}\right)}{2 K\left[\left(1+f y_{*}\right)\left(x_{*}+A\right)\right]^{2}}, \\
& c_{12}=\frac{f r x_{*}\left(2 K-3 x_{*}\right)\left(x_{*}+A\right)^{2}+f r x_{*}^{2}\left(x_{*}-K\right)\left(x_{*}+A\right)}{6 K\left[\left(1+f y_{*}\right)\left(x_{*}+A\right)\right]^{3}}, \\
& c_{03}=\frac{6 r x_{*}^{2} f^{3}\left(x_{*}-K\right)}{K\left(x_{*}+A\right)\left(1+f y_{*}\right)^{4}}, \\
& d_{10}=\text { cay }_{*}, \\
& d_{01}=0 \text {, } \\
& d_{20}=0 \text {, } \\
& d_{11}=\frac{c a}{2}, \\
& d_{02}=0 \text {, } \\
& d_{30}=0 \text {, } \\
& d_{21}=0 \text {, } \\
& d_{12}=0 \text {, } \\
& d_{03}=0 \text {. }
\end{aligned}
$$


Journal of Mathematics

7

Let

$$
\begin{aligned}
P_{1}(X, Y)= & c_{20} X^{2}+c_{11} X Y+c_{02} Y^{2}+c_{30} X^{3} \\
& +c_{21} X^{2} Y+c_{12} X Y^{2}+c_{03} Y^{3}, \\
P_{2}(X, Y)= & d_{20} X^{2}+d_{11} X Y+d_{02} Y^{2}+d_{30} X^{3} \\
& +d_{21} X^{2} Y+d_{12} X Y^{2}+d_{03} Y^{3} .
\end{aligned}
$$

Now,

$$
\begin{aligned}
& \dot{X}=c_{10} X+c_{01} Y+P_{1}(X, Y), \\
& \dot{Y}=d_{10} X+d_{01} Y+P_{2}(X, Y) .
\end{aligned}
$$

Taking the transformation $u=X$ and $v=-(1 / \beta)$ $\left(c_{10} X+c_{01} Y\right)$, where

$$
\begin{aligned}
G_{1}(u, v)= & P_{1}\left(u,-\frac{1}{c_{01}}\left(c_{10} u+\beta v\right)\right) \\
G_{2}(u, v)= & -\frac{1}{\beta}\left(c_{10} P_{1}\left(u,-\frac{1}{c_{01}}\left(c_{10} u+\beta v\right)\right)\right) \\
& +c_{01} P_{2}\left(u,-\frac{1}{c_{01}}\left(c_{10} u+\beta v\right)\right) .
\end{aligned}
$$

Therefore,

$$
\begin{aligned}
& \frac{\mathrm{d} u}{\mathrm{~d} t}=-\beta v+G_{1}(u, v), \\
& \frac{\mathrm{d} v}{\mathrm{~d} t}=\beta u+G_{2}(u, v),
\end{aligned}
$$

$$
\begin{aligned}
G_{1}(u, v)= & c_{20} u^{2}+c_{11} u\left(-\frac{1}{c_{01}}\left(c_{10} u+\beta v\right)\right)+c_{02}\left(-\frac{1}{c_{01}}\left(c_{10} u+\beta v\right)\right)^{2}+c_{30} u^{3}+ \\
& c_{21} u^{2}\left(-\frac{1}{c_{01}}\left(c_{10} u+\beta v\right)\right)+c_{12} u\left(-\frac{1}{c_{01}}\left(c_{10} u+\beta v\right)\right)^{2}+c_{03}\left(-\frac{1}{c_{01}}\left(c_{10} u+\beta v\right)\right)^{3}, \\
G_{2}(u, v)= & -\frac{1}{\beta}\left[c _ { 1 0 } \left(c_{20} u^{2}+c_{11} u\left(-\frac{1}{c_{01}}\left(c_{10} u+\beta v\right)\right)+c_{02}\left(-\frac{1}{c_{01}}\left(c_{10} u+\beta v\right)\right)^{2}+c_{30} u^{3}\right.\right. \\
& \left.\left.+c_{21} u^{2}\left(-\frac{1}{c_{01}}\left(c_{10} u+\beta v\right)\right)+c_{12} u\left(-\frac{1}{c_{01}}\left(c_{10} u+\beta v\right)\right)^{2}+c_{03}\left(-\frac{1}{c_{01}}\left(c_{10} u+\beta v\right)\right)^{3}\right)\right] \\
& +c_{01} d_{11} u\left(-\frac{1}{c_{01}}\left(c_{10} u+\beta v\right)\right) .
\end{aligned}
$$

The following is set:

$$
\begin{aligned}
\psi= & \frac{1}{16}\left[\frac{\partial^{3} G_{1}}{\partial u^{3}}+\frac{\partial^{3} G_{1}}{\partial u \partial v^{2}}+\frac{\partial^{3} G_{2}}{\partial u^{2} \partial v}+\frac{\partial^{3} G_{2}}{\partial v^{3}}\right]+\frac{1}{16 \beta}\left[\frac{\partial^{2} G_{1}}{\partial u \partial v}\left(\frac{\partial^{2} G_{1}}{\partial u^{2}}+\frac{\partial^{2} G_{1}}{\partial v^{2}}\right)\right. \\
& \left.-\frac{\partial^{2} G_{2}}{\partial u \partial v}\left(\frac{\partial^{2} G_{2}}{\partial u^{2}}+\frac{\partial^{2} G_{2}}{\partial v^{2}}\right)-\frac{\partial^{2} G_{1}}{\partial u^{2}} \frac{\partial^{2} G_{2}}{\partial u^{2}}+\frac{\partial^{2} G_{1}}{\partial v^{2}} \frac{\partial^{2} G_{2}}{\partial v^{2}}\right]
\end{aligned}
$$

where 


$$
\begin{aligned}
& \frac{\partial^{3} G_{1}}{\partial u^{3}}=6\left(c_{30}-\frac{c_{10} c_{21}}{c_{01}}+\frac{c_{10}^{2} c_{12}}{c_{01}^{2}}-\frac{c_{10}^{3} c_{03}}{c_{01}^{3}}\right) \\
& \frac{\partial^{3} G_{1}}{\partial u \partial v^{2}}=2 \beta^{2}\left(\frac{c_{12}}{c_{01}^{2}}-\frac{2 c_{10} c_{03}}{c_{01}^{3}}-\frac{c_{10} c_{03}}{c_{01}^{3}}\right) \\
& \frac{\partial^{2} G_{1}}{\partial u \partial v}=\beta\left(\frac{2 c_{10} c_{02}}{c_{01}^{2}}-\frac{c_{11}}{c_{01}}\right)-2 \beta u\left(\frac{c_{21}}{c_{01}}-\frac{2 c_{10} c_{12}}{c_{01}^{2}}+\frac{c_{10}^{2} c_{03}}{c_{01}^{3}}+\frac{2 c_{10}^{2} c_{03}}{c_{01}^{3}}\right) \\
& +2 \beta^{2} v\left(\frac{c_{12}}{c_{01}^{2}}-\frac{2 c_{10} c_{03}}{c_{01}^{3}}-\frac{c_{10} c_{03}}{c_{01}^{3}}\right) \\
& \frac{\partial^{2} G_{1}}{\partial u^{2}}=2\left(c_{20}-\frac{c_{10} c_{11}}{c_{01}}+\frac{c_{10}^{2} c_{02}}{c_{01}^{2}}\right)+6 u\left(c_{30}-\frac{c_{10} c_{21}}{c_{01}}+\frac{c_{10}^{2} c_{12}}{c_{01}^{2}}-\frac{c_{10}^{3} c_{03}}{c_{01}^{3}}\right) \\
& +2 \beta v\left(\frac{2 c_{10} c_{12}}{c_{01}^{2}}-\frac{c_{21}}{c_{01}}-\frac{c_{10}^{2} c_{03}}{c_{01}^{3}}-\frac{2 c_{10}^{2} c_{03}}{c_{01}^{3}}\right) \\
& \frac{\partial^{2} G_{1}}{\partial v^{2}}=2 \beta^{2} u\left(\frac{c_{12}}{c_{01}^{2}}-\frac{2 c_{10} c_{03}}{c_{01}^{3}}-\frac{c_{10} c_{03}}{c_{01}^{3}}\right)+\frac{2 c_{02}}{c_{01}^{2}} \beta^{2}-6 \frac{c_{03}}{c_{01}^{3}} \beta^{3} v \\
& \frac{\partial^{3} G_{2}}{\partial v^{3}}=6 \frac{c_{10} c_{03}}{c_{01}^{3}} \beta^{2} \\
& \frac{\partial^{3} G_{2}}{\partial u^{2} \partial v}=2\left(\frac{c_{10} c_{21}}{c_{01}}-\frac{2 c_{10}^{2} c_{12}}{c_{01}^{2}}+\frac{c_{10}^{3} c_{03}}{c_{01}^{3}}+\frac{2 c_{10}^{3} c_{03}}{c_{01}^{3}}\right), \\
& \frac{\partial^{2} G_{2}}{\partial u \partial v}=2 u\left(\frac{c_{10} c_{21}}{c_{01}}-\frac{2 c_{10}^{2} c_{12}}{c_{01}^{2}}+\frac{c_{10}^{3} c_{03}}{c_{01}^{3}}+\frac{2 c_{10}^{3} c_{03}}{c_{01}^{3}}\right) \\
& +2 \beta v\left(\frac{c_{10}^{2} c_{03}}{c_{01}^{3}}-\frac{c_{10} c_{12}}{c_{01}^{2}}+\frac{2 c_{10}^{2} c_{03}}{c_{01}^{3}}\right)+\frac{c_{10} c_{11}}{c_{01}}-2 \frac{c_{10}^{2} c_{02}}{c_{01}^{2}}-d_{11} \beta, \\
& \frac{\partial^{2} G_{2}}{\partial u^{2}}=6 u\left(\frac{c_{10}^{2} c_{21}}{c_{01} \beta}-\frac{c_{10} c_{30}}{\beta}-\frac{c_{10}^{3} c_{12}}{c_{01}^{2} \beta}+\frac{c_{10}^{4} c_{03}}{c_{01}^{3} \beta}\right)+2 v\left(\frac{c_{10} c_{21}}{c_{01}}-\frac{2 c_{10}^{2} c_{12}}{c_{01}^{2}}+\frac{c_{10}^{3} c_{03}}{c_{01}^{3}}+\frac{2 c_{10}^{3} c_{03}}{c_{01}^{3}}\right) \\
& +2\left(\frac{c_{10}^{2} c_{11}}{c_{01} \beta}-\frac{c_{10} c_{20}}{\beta}-\frac{c_{10}^{3} c_{02}}{c_{01}^{2} \beta}\right)-c_{10} d_{11} \\
& \frac{\partial^{2} G_{2}}{\partial v^{2}}=2 \beta u\left(\frac{c_{10}^{2} c_{03}}{c_{01}^{3}}-\frac{c_{10} c_{12}}{c_{01}^{2}}+\frac{2 c_{10}^{2} c_{03}}{c_{01}^{3}}\right)-2 \frac{c_{10} c_{02}}{c_{01}^{2}} \beta+6 \frac{c_{10} c_{03}}{c_{01}^{3}} \beta^{2} v
\end{aligned}
$$

If $\psi<0$, the Hopf bifurcation is supercritical, and the Hopf bifurcation is subcritical if $\psi>0$. 


\section{Stability and Hopf Bifurcation Analysis of the Delay Model}

Letting $X(t)=x(t)-x_{*}$ and $Y(t)=y(t)-y_{*}$, after linearization, model (6) could be expressed as the following matrix:

$$
\frac{\mathrm{d}}{\mathrm{d} t}\left(\begin{array}{c}
X(t) \\
Y(t)
\end{array}\right)=A_{1}\left(\begin{array}{c}
X(t) \\
Y(t)
\end{array}\right)+A_{2}\left(\begin{array}{c}
x(t-\tau) \\
y(t-\tau)
\end{array}\right),
$$

where

$$
\begin{aligned}
A_{1} & =\left(\begin{array}{cc}
\frac{2 r x_{*}\left(1+f y_{*}\right)\left(x_{*}+A\right)-r x_{*}^{2} K\left(1+f y_{*}\right)}{\left[\left(1+f y_{*}\right)\left(x_{*}+A\right)\right]^{2}} & \frac{-r f x_{*}^{2}\left(x_{*}+A\right)}{\left[\left(1+f y_{*}\right)\left(x_{*}+A\right)\right]^{2}} \\
-\frac{2 r x_{*}^{2} K\left(1+f y_{*}\right)\left(x_{*}+A\right)-r x_{*}^{3}\left(1+f y_{*}\right)}{\left[K\left(1+f y_{*}\right)\left(x_{*}+A\right)\right]^{2}}-a y_{*} & +\frac{r K f x_{*}^{2}\left(x_{*}+A\right)}{\left[k\left(1+f y_{*}\right)\left(x_{*}+A\right)\right]^{2}}-\frac{\delta}{c} \\
c a y_{*} & 0
\end{array}\right), \\
A_{2} & =\left(\begin{array}{c}
-\frac{r x_{*}^{2}}{k\left(1+f y_{*}\right)\left(x_{*}+A\right)} \\
0 \\
0
\end{array}\right) .
\end{aligned}
$$

Let

$$
\begin{aligned}
G= & \frac{2 r x_{*}\left(1+f y_{*}\right)\left(x_{*}+A\right)-r x_{*}^{2} K\left(1+f y_{*}\right)}{\left[\left(1+f y_{*}\right)\left(x_{*}+A\right)\right]^{2}} \\
& -\frac{2 r x_{*}^{2} K\left(1+f y_{*}\right)\left(x_{*}+A\right)-r x_{*}^{3}\left(1+f y_{*}\right)}{\left[K\left(1+f y_{*}\right)\left(x_{*}+A\right)\right]^{2}}-a y_{*},
\end{aligned}
$$$$
H=\frac{r x_{*}^{2}}{k\left(1+f y_{*}\right)\left(x_{*}+A\right)}
$$$$
I=\frac{r K f x_{*}^{2}\left(x_{*}+A\right)}{\left[k\left(1+f y_{*}\right)\left(x_{*}+A\right)\right]^{2}}-\frac{r f x_{*}^{2}\left(x_{*}+A\right)}{\left[\left(1+f y_{*}\right)\left(x_{*}+A\right)\right]^{2}} .
$$

The characteristic polynomial is

$$
H(\lambda)=\lambda^{2}-\left(G-H e^{-\lambda t}\right) \lambda+a \delta y_{*}-I c a y_{*} .
$$

Theorem 4. If $H^{2}-G^{2}+2 F-2 I L>0$ and $-F^{2}+2 I F L-I^{2} L^{2}>0$, the interior equilibrium $E_{*}$ is locally asymptotically stable, and Hopf bifurcation occurs when $\tau$ passes the value

$$
\bar{\tau}=\frac{1}{\omega_{0}} \arctan \frac{\omega_{0}^{2}+I L-F}{G \omega_{0}} .
$$

Proof. The characteristic equation is

$$
\lambda^{2}-\left(G-H e^{-\lambda t}\right) \lambda+a \delta y_{*}-I_{c a y}=0 .
$$

Supposing that $\lambda\left(\tau_{0}\right)=i \omega_{0}$ is a solution of $H(\lambda)$, one has

$$
-\omega_{0}^{2}-\left(G-H e^{-i \omega_{0} \tau}\right) i \omega_{0}+a \delta y_{*}-I c a y_{*}=0 .
$$

It is known that

$$
e^{-\omega_{0} \tau}=\cos \omega_{0} \tau-i \sin \omega_{0} \tau,
$$

so one obtains

$-\omega_{0}^{2}-\left(G-H\left(\cos \omega_{0} \tau-i \sin \omega_{0} \tau\right)\right) i \omega_{0}+a \delta y_{*}-I c a y_{*}=0$.

Let $F=a \delta y_{*}$ and $L=I c a y_{*}$; then,

$-\omega_{0}^{2}-i \omega_{0} G+i \omega_{0} H \cos \omega_{0} \tau+H \omega_{0} \sin \omega_{0} \tau+F-I L=0$.

Next, separating real and imaginary parts,

$$
\begin{aligned}
H \omega_{0} \sin \omega_{0} \tau & =\omega_{0}^{2}+I L-F, \\
\omega_{0} H \cos \omega_{0} \tau & =\omega_{0} G,
\end{aligned}
$$

one obtains

$$
\omega_{0}^{4}+\omega_{0}^{2}\left(H^{2}-G^{2}+2 F-2 I L\right)-F^{2}+2 I F L-I^{2} L^{2}=0 .
$$

Assuming that

$$
W=\omega_{0}^{2}
$$

one has 


$$
W^{2}+W\left(H^{2}-G^{2}+2 F-2 I L\right)-F^{2}+2 I F L-I^{2} L^{2}=0 .
$$

If $H^{2}-G^{2}+2 F-2 I L>0$ and $-F^{2}+2 I F L-I^{2} L^{2}>0$, all roots of the equation have negative real parts, which means that the interior equilibrium $E_{*}$ is locally asymptotically stable:

$$
W=\frac{-N \pm \sqrt{N^{2}-4\left(-F^{2}+2 I F L-I^{2} L^{2}\right)}}{2},
$$

where

$$
N=H^{2}-G^{2}+2 F-2 I L .
$$

If $-N>0, N^{2}=4\left(-F^{2}+2 I F L-I^{2} L^{2}\right)$, and $-F^{2}+2 I F L$ $-I^{2} L^{2}>0$, the equation has a unique positive solution. The interior equilibrium $E_{*}$ is stable. If $-N>0, N^{2}>4$ $\left(-F^{2}+2 I F L-I^{2} L^{2}\right)$, and $-F^{2}+2 I F L-I^{2} L^{2}>0$, the equation has two positive solutions.

One has

$$
\begin{aligned}
H \omega_{0} \sin \omega_{0} \tau & =\omega_{0}^{2}+I L-F, \\
\omega_{0} H \cos \omega_{0} \tau & =\omega_{0} G,
\end{aligned}
$$

so

$$
\begin{aligned}
& \sin \omega_{0} \tau=\frac{\omega_{0}^{2}+I L-F}{H \omega_{0}}, \\
& \cos \omega_{0} \tau=\frac{G}{H},
\end{aligned}
$$

and one obtains

$$
\tan \omega_{0} \tau=\frac{\omega_{0}^{2}+I L-F}{G \omega_{0}} .
$$

This shows that if $-N<0$ and $-F^{2}+2 I F L-I^{2} L^{2}<0$, the characteristic equation

$$
\lambda^{2}-\left(G-H e^{-\lambda t}\right) \lambda+a \delta y_{*}-I c a y_{*}=0
$$

has a pair of imaginary eigenvalues $\lambda= \pm i \omega_{0}, \eta(\bar{\tau})=0$ when $\bar{\tau}_{j}^{ \pm}=\left(1 / \omega_{0}^{ \pm}\right) \arctan \left(\omega_{0}^{2}+I L-F / G \omega_{0}\right)+\left(2 \pi j / \omega_{0}^{ \pm}\right), j$ $=0,1, \ldots$.

Next, the cross-sectional condition holds and is proved.

The characteristic equation is

$$
\lambda^{2}-\left(G-H e^{-\lambda t}\right) \lambda+F-I L=0
$$

at $j=0$ and $\tau=\bar{\tau}=\bar{\tau}_{j}$.

One obtains

$$
\begin{aligned}
2 \lambda \frac{\mathrm{d} \lambda}{\mathrm{d} \tau}-\frac{\mathrm{d} \lambda}{\mathrm{d} \tau}\left(G-H e^{-\lambda \tau}\right)+\lambda G e^{-\lambda \tau}\left(-\tau \frac{\mathrm{d} \lambda}{\mathrm{d} \tau}-\lambda\right) & =0 \\
\left(\frac{\mathrm{d} \lambda}{\mathrm{d} \tau}\right)^{-1} & =\frac{\tau}{\lambda}-\frac{H}{\lambda^{2} G}+\frac{1}{\lambda^{2} e^{-\lambda \tau}} \\
& -\frac{2}{\lambda G e^{-\lambda \tau}} \\
\left(\frac{\mathrm{d} \operatorname{Re}(\lambda)}{\mathrm{d} \tau}\right)^{-1} & \neq 0 .
\end{aligned}
$$

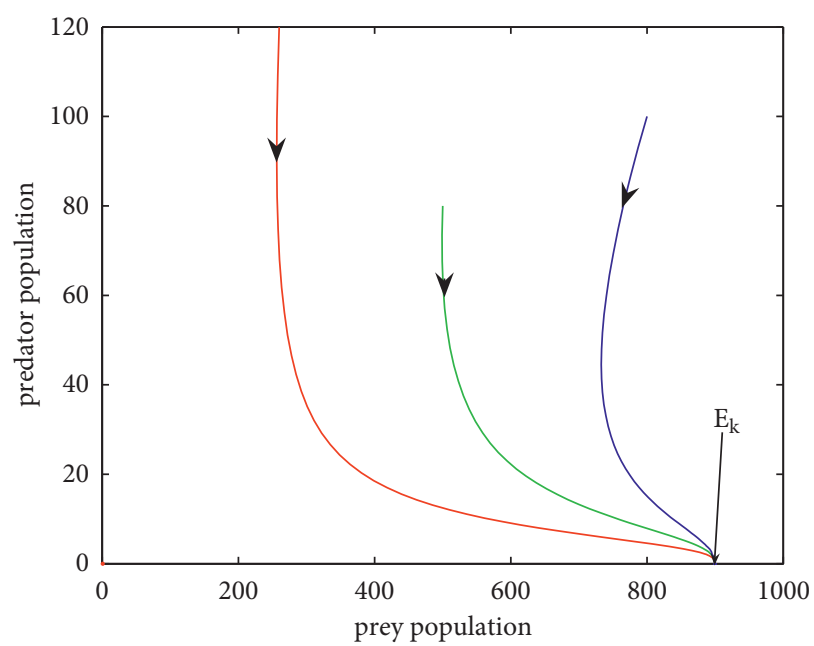

Figure 2: Phase portrait of model (5); $E_{K}$ is stable $(r=2.65, f=0.1$, $K=900, A=5, a=0.002, c=0.215$, and $\delta=1.06$ ).

\section{Numerical Simulations}

In this section, the realization of numerical simulation uses Matlab 2016a (MathWorks, USA). Through extensive numerical simulations, several results that verify the above analytical analysis conclusion of models (5) and (6) are obtained. The parameters are fixed as $r=2.65, f=0.1$, $K=900, c=0.215$, and $\delta=1.06$, and $a, A$, and $\tau$ are considered controlling parameters. Letting $a=0.002<(\delta / c K)$, $E_{K}$ is stable. Considering the parameters $a=0.02$ and $A=5$, which satisfy the conditions of Theorem 2, the interior equilibrium $E_{*}(246.5,26.4)$ is locally asymptotically stable. Let $A=149$, the other parameters are unchanged, and the interior equilibrium becomes unstable. A series of calculations show that $\bar{\tau}=7.752$, and $E_{*}$ loses its stability when $\tau$ crosses through the critical $\bar{\tau}$.

First, the stability conditions of $E_{K}$ and $E_{*}$ are obtained. By choosing appropriate parameters for the numerical simulations, the stable phase portraits of $E_{K}$ and $E_{*}$ are obtained, as shown in Figures 2 and 3. The stable phase portrait of $E_{*}$ without the Allee effect is also obtained (Figure 4, $A=0$ ). By comparing Figures $4-6$, it is found that the stability of the equilibrium point $E_{*}$ will change as parameter $A$ changes. A bifurcation diagram is provided, so the results look more intuitive. As Figure 7 shows, a bifurcation occurs when the bifurcation parameter value is approximately 65 , which is consistent with this study. The population dynamic changes from stable to unstable with an increasing value of parameter A. Finally, the dynamical behaviors of model (6) are studied, and mainly, the effect of gestation delay on the stability of the interior equilibrium point is simulated. Figures 8 and 9 show that the equilibrium point is stable at $\tau=0.9$ and unstable at $\tau=8$. In Figure 10, when $f=0$, the other parameters remain unchanged, and the model is simulated without the fear effect. The phase portrait is unstable and shows periodicity with increasing time $(\tau=0.9)$. Upon comparison with Figure 8, the existence of the fear effect promotes the stability of the phase portrait. 


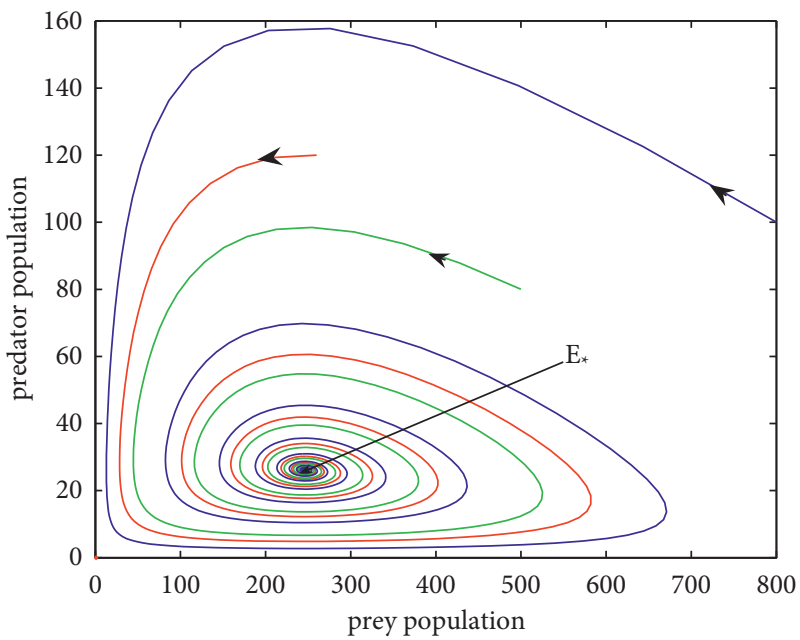

Figure 3: Phase portrait of model (5); $E_{*}$ is stable $(r=2.65, f=0.1, K=900, A=5, a=0.02, c=0.215$, and $\delta=1.06)$.

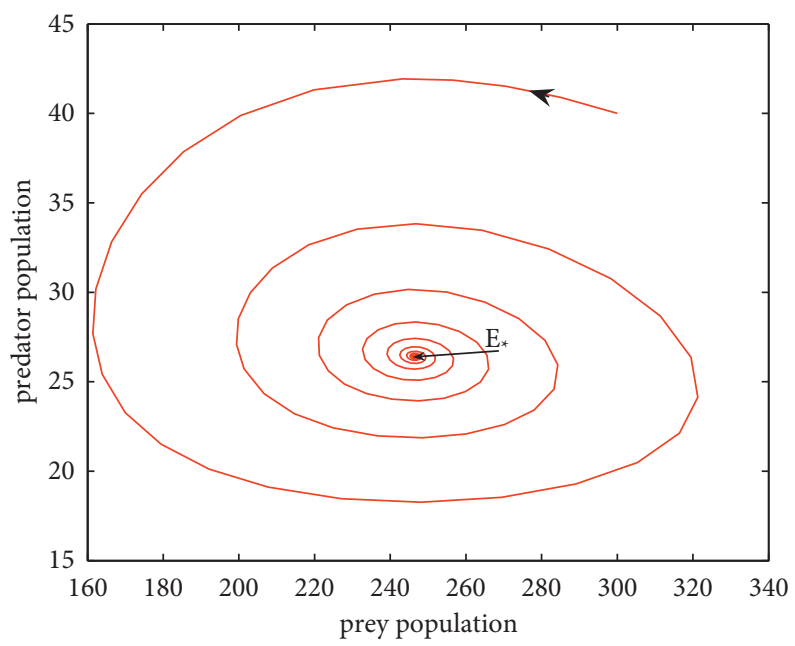

Figure 4: Phase portrait of model (5); $E_{*}$ is stable $(r=2.65, f=0.1, K=900, A=0, a=0.02, c=0.215$, and $\delta=1.06)$.

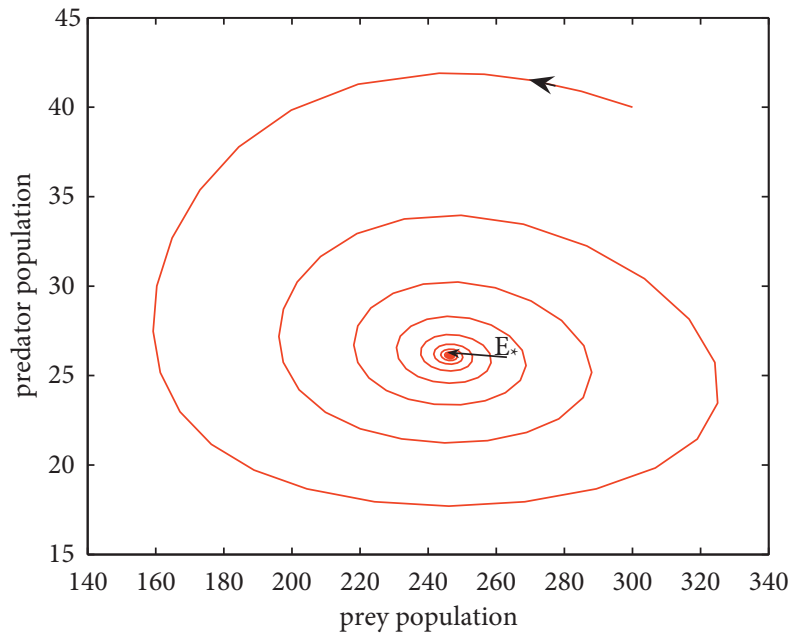

Figure 5: Phase portrait of model (5); $E_{*}$ is stable $(r=2.65, f=0.1, K=900, A=5, a=0.02, c=0.215$, and $\delta=1.06)$. 


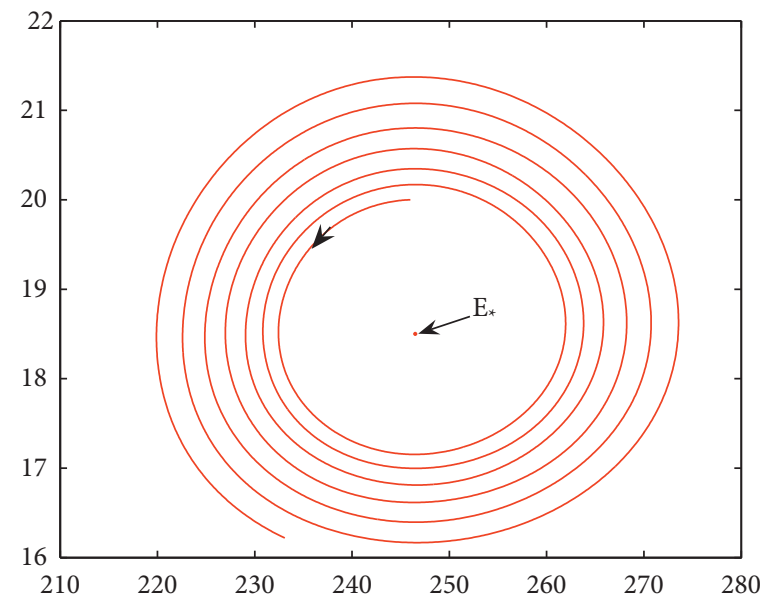

Figure 6: Phase portrait of model (5); $E_{*}$ is unstable $(r=2.65, f=0.1, K=900, A=149, a=0.02, c=0.215$, and $\delta=1.06)$.

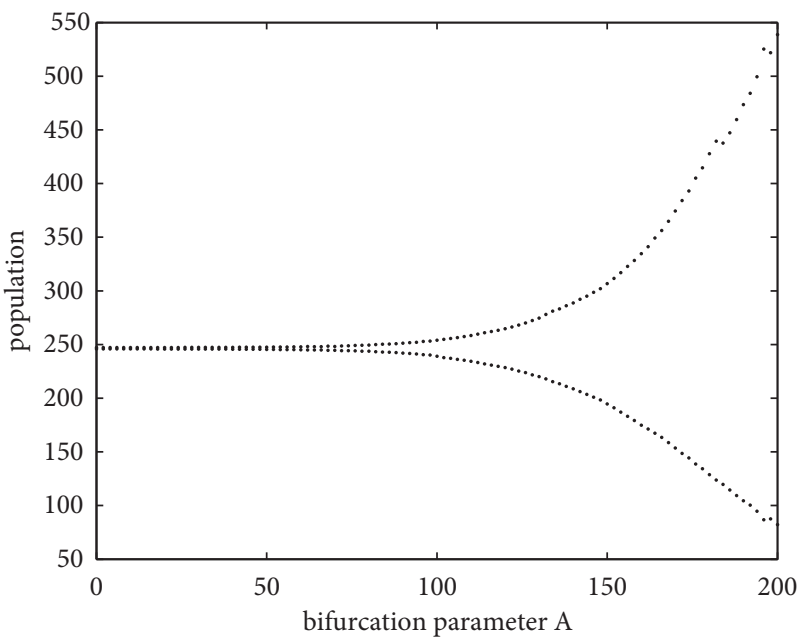

Figure 7: Bifurcation diagram with parameter $A(r=2.65, f=0.1, K=900, a=0.02, c=0.215$, and $\delta=1.06)$.

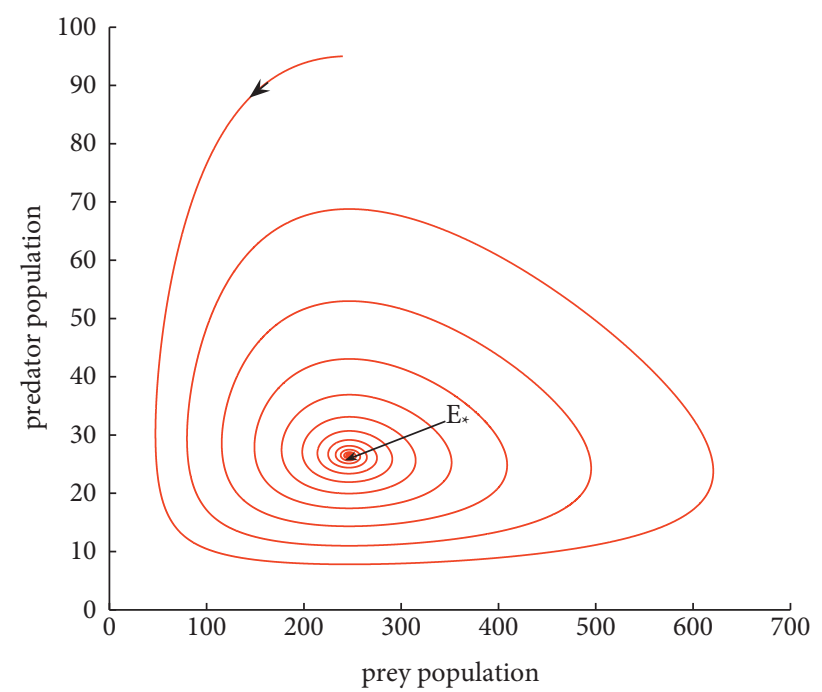

Figure 8: Phase portrait of model (6); $E_{*}$ is stable $(r=2.65, f=0.1, K=900, A=0.01, a=0.02, c=0.215, \delta=1.06$, and $\tau=0.9)$. 


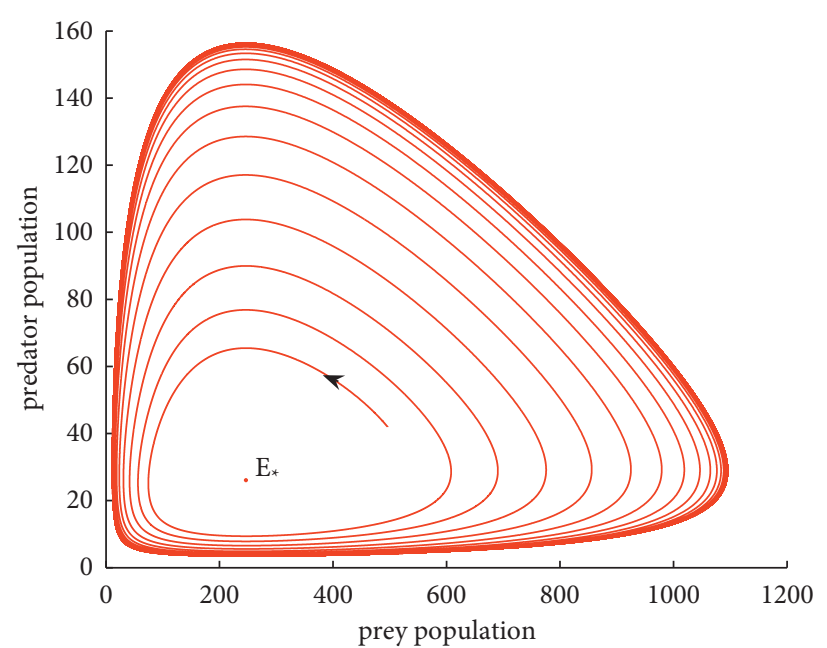

Figure 9: Phase portrait of model (6); $E_{*}$ is unstable $(r=2.65, f=0.1, K=900, A=0.01, a=0.02, c=0.215, \delta=1.06$, and $\tau=8)$.

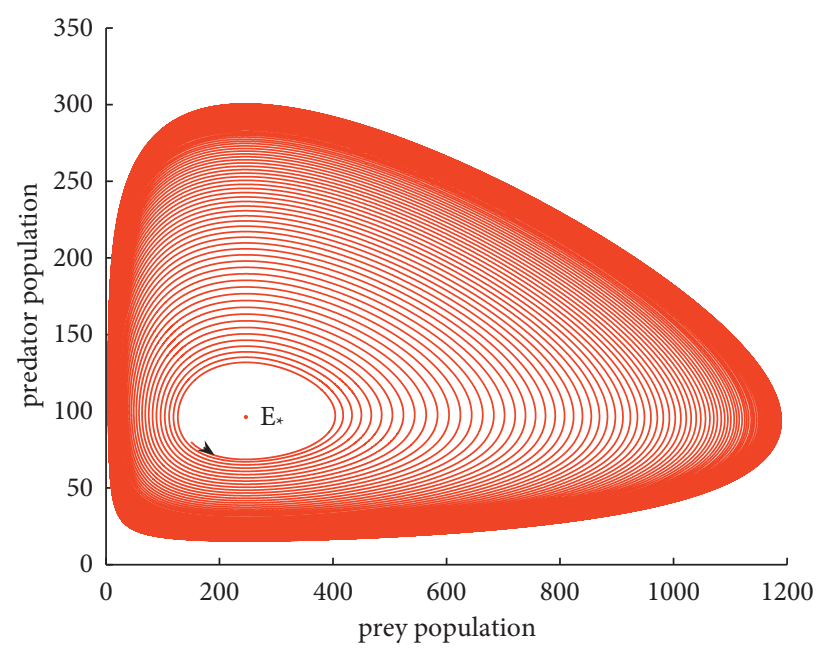

Figure 10: Phase portrait of model (6); $E_{*}$ is unstable $(r=2.65, f=0, K=900, A=0.01, a=0.02, c=0.215, \delta=1.06$, and $\tau=0.9)$.

\section{Conclusions}

Mathematical models play an important role in many fields, such as ecology [29-31], dynamics [32], physics [33], algorithms [34, 35], and epidemiology [36-38]. In this study, a predator-prey model is established with a fear effect and weak Allee effect, and the effect of the weak Allee effect on the model is studied. All equilibrium points of the model were solved, and stability analysis was performed. Furthermore, the conditions required for the equilibrium points to be stable were determined. The boundedness and stability of the model were analyzed. Model (5), which undergoes a transcritical bifurcation at the axial equilibrium point $E_{K}$ and undergoes a Hopf bifurcation at the interior equilibrium point $E_{*}$, was also analyzed. Moreover, the direction of the Hopf bifurcation was determined. After completing these proofs, gestation delay was introduced into model (5) to obtain model (6). Time delay can change the stability of model (6); that is, the stability of the interior equilibrium point of model (6) changes with the change in delay. Finally, parameters were selected that satisfy the conditions for numerical simulation. Stable phase portraits of the equilibrium points $E_{K}$ and $E_{*}$ were obtained. By increasing the value of parameter $A$, it was found that the stability of the equilibrium point $E_{*}$ changed. Upon comparison with the situation without the Allee effect, it was found that the Allee effect has a significant impact on the stability of the equilibrium point. Then, phase portraits and a bifurcation diagram with $A$ as the parameter were generated to verify the study. The effect of gestation delay on the stability of the interior equilibrium point was mainly studied using model (6). By giving unstable and stable phase portraits, the gestation delay will change the stability of the original model. When the model has no fear effect, the stable phase portrait becomes unstable. In other words, the fear effect promotes stability.

Dynamic complexities are the common characteristics in a variety of systems. The complexity of an ecosystem is reflected in both time and space $[39,40]$. From a biological point of view, this research shows that a weak Allee effect and gestation delay can destroy the stability of a species and lead to a decrease in population density. Moreover, the 
introduction of some ecological effects makes the model closer to representing the complex relationships between populations. Appropriate model construction can capture accurate characteristics of interacting population dynamics. This is an important modeling idea to help us better understand population dynamics. Generally speaking, considering the weak Allee effect, fear effect, and gestation delay, the model proposed in this study is more biologically reasonable than some previous predator-prey models. Our finding suggests that the Allee effect and delay can cause unexpected dynamic predictions of interacting populations, such as stability switching. This result is more or less similar to some previous viewpoints. Ye et al. [4] studied the dynamic behavior of a predator-prey model and found that the Allee effect, searching delay, and digestion delay can all cause Hopf bifurcation. Huang et al. [41] showed that the Allee effect, fear effect, and prey refuge will complicate the dynamic behavior of a predator-prey model. Another important result is that the stability of the model with weak Allee effect and gestation delay is very sensitive to changes in the fear level. Therefore, the model with a Holling type-I functional response and weak Allee effect makes it difficult to resist changes in the fear effect. Hence, a low level of fear will have a significant impact on the stability of the model. Overall, it is found that a weak Allee effect, fear effect, and delay all play significant roles in population dynamics. The previous studies show that the Allee effect and delay can induce Hopf bifurcation and that the fear effect will promote the system stability $[4,22,42,43]$. Wang et al. [11] considered the fear effect term in the interacting prey-predator model and completed a comprehensive work on fear factor. Qiao et al. [21] showed that the fear effect reduces the population density of prey and causes Hopf bifurcation. Overall, these findings suggest that a weak Allee effect, fear effect, and delay all play significant roles in population dynamics. According to the existing references, the present work covers a variety of ecological effects and obtained appropriate results.

\section{Data Availability}

The data used to support the findings of this study are included within the article.

\section{Conflicts of Interest}

The authors declare that they have no conflicts of interest.

\section{Authors' Contributions}

All of the authors contributed equally and significantly to this study. All read and approved the final manuscript.

\section{Acknowledgments}

All the authors thank Northwest Minzu University for supporting this work. This work was supported by the Gansu Science and Technology Fund (20JR5RA512), Research Fund for Humanities and Social Sciences of the Ministry of Education (20XJAZH006), the Leading Talents Project of
State Ethnic Affairs Commission of China, Central Universities Fundamental Research Funds for the Graduate Students of Northwest Minzu University (Yxm2021067), and Innovation Team of Intelligent Computing and Dynamical System Analysis and Application of Northwest Minzu University.

\section{References}

[1] A. J. Lotka, Elements of Physical Biology, Williams and Wilkins, Pennsylvania, PA, USA, 1926.

[2] V. Volterra, "Fluctuations in the abundance of a species considered mathematically," Nature, vol. 118, no. 2972, pp. 558-560, 1926.

[3] B. Chakraborty, H. Baek, and N. Bairagi, "Diffusion-induced regular and chaotic patterns in a ratio-dependent predator-prey model with fear factor and prey refuge," Chaos, vol. 31, Article ID 033128, 2021.

[4] Y. Ye, H. Liu, Y. Wei, M. Ma, and K. Zhang, "Dynamic study of a predator-prey model with weak Allee effect and delay," Advances in Mathematical Physics, vol. 2019, Article ID 7296461, 2019.

[5] H. Liu, Y. Ye, Y. Wei, M. Ma, and J. Ye, "Dynamic study of a predator-prey system with weak Allee effect and holling typeIII functional response," Dynamic Systems and Applications, vol. 27, no. 4, pp. 943-953, 2018.

[6] L. Y. Zanette, A. F. White, M. C. Allen, and M. Clinchy, "Perceived predation risk reduces the number of offspring songbirds produce per year," Science, vol. 334, no. 6061, pp. 1398-1401, 2011.

[7] W. Cresswell, "Predation in bird populations," Journal of Ornithology, 2011.

[8] E. L. Preisser and D. I. Bolnick, "The many faces of fear: comparing the pathways and impacts of nonconsumptive predator effects on prey populations," PLoS One, vol. 3, no. 6, 2008.

[9] S. Creel and D. Christianson, "Relationships between direct predation and risk effects," Trends in Ecology \& Evolution, vol. 23, no. 4, pp. 194-201, 2008.

[10] M. G. Pretelli, J. P. Isacch, and D. A. Cardoni, "Variation in parental care in the spectacled tyrant hymenops perspicillatus is associated with increased nest predation in grassland fragments," Journal of Ornithology, vol. 157, no. 2, pp. 451-460, 2016.

[11] X. Wang, L. Zanette, and X. Zou, "Modelling the fear effect in predator-prey interactions," Journal of Mathematical Biology, vol. 73, 2016.

[12] X. Wang and X. Zou, "Modeling the fear effect in predatorprey interactions with adaptive avoidance of predators," Bulletin of Mathematical Biology, vol. 79, no. 6, pp. 1325-1359, 2017.

[13] F. Courchamp, T. Clutton-Brock, and B. Grenfell, "Inverse density dependence and the allee effect," Trends in Ecology \& Evolution, vol. 14, no. 10, pp. 405-410, 1999.

[14] P. A. Stephens, W. J. Sutherland, and R. P. Freckleton, "What is the allee effect?" Oikos, vol. 87, no. 1, pp. 185-190, 1999.

[15] P. A. Stephens and W. J. Sutherland, "Consequences of the Allee effect for behaviour, ecology and conservation," Trends in Ecology \& Evolution, vol. 14, no. 10, pp. 401-405, 1999.

[16] A. Deredec and F. Courchamp, "Extinction thresholds in host-parasite dynamics," Annales Zoologici Fennici, vol. 40, pp. 115-130, 2003.

[17] M. H. Wang and M. Kot, "Speeds of invasion in a model with strong or weak allee effects," Mathematical Biosciences, vol. 171, no. 1, pp. 83-97, 2001. 
[18] C. W. Fowler and J. D. Baker, "A review of animal population dynamics at extremely reduced population levels," Report International Whaling Commission, vol. 41, pp. 545-554, 1991.

[19] S. J. Schreiber, "Allee effects, extinctions, and chaotic transients in simple population models," Theoretical Population Biology, vol. 64, no. 2, pp. 201-209, 2003.

[20] R. Lin, S. Liu, and X. Lai, "Bifurcations of a predator-prey system with weak allee effects," Journal of the Korean Mathematical Society, vol. 50, no. 4, pp. 695-713, 2013.

[21] T. Qiao, Y. Cai, S. Fu, and W. Wang, "Stability and hopf bifurcation in a predator-prey model with the cost of antipredator behaviors," International Journal of Bifurcation and Chaos, vol. 29, no. 13, 2019.

[22] R. Yang, H. Ren, and X. Cheng, "A diffusive predator-prey system with prey refuge and gestation delay," Advances in Difference Equations, vol. 158, 2017.

[23] B. Mukhopadhyay and R. Bhattacharyya, "Role of gestation delay in a plankton-fish model under stochastic fluctuations," Mathematical Biosciences, vol. 215, no. 1, pp. 26-34, 2008.

[24] O. Kolade, K. Berat, and B. Dumitru, "Pattern formation in superdiffusion predatorcprey-like problems with integer- and noninteger-order derivatives," Mathematical Methods in the Applied Sciences, vol. 2020, pp. 1-19, 2020.

[25] O. Kolade, "Computational dynamics of predator-prey model with the power-law kernel," Results in Physics, vol. 21, Article ID 103810, 2021.

[26] K. Zhang, X. Wang, H. Liu et al., "Mathematical analysis of a human papillomavirus transmission model with vaccination and screening," Mathematical Biosciences and Engineering, vol. 5, $20202646 \mathrm{C} 2670$.

[27] Q. Jiang, J. Xie, and J. Ye, Mathematical Model, Higher Education Press, Beijing, China, 2001.

[28] Perko, Different Equations and Dynamical Systems, Springer, Berlin, Germany, 2001.

[29] S. Kumar, R. Kumar, C. Cattani, and B. Samet, "Chaotic behaviour of fractional predator-prey dynamical system," Chaos, Solitons \& Fractals, vol. 135, Article ID 109811, 2020.

[30] S. Kumar, R. Kumar, R. P. Agarwal, and B. Samet, “A study of fractional lotka-volterra population model using haar wavelet and adams-bashforth-moulton methods," Mathematical Methods in the Applied Sciences, vol. 2020, pp. 1-15, 2020.

[31] B. Ghanbari, S. Kumar, and R. Kumar, "A study of behaviour for immune and tumor cells in immunogenetic tumour model with non-singular fractional derivative," Chaos, Solitons \& Fractals, vol. 133, Article ID 109619, 2020.

[32] E. F. Doungmo Goufo, S. Kumar, and S. B. Mugisha, "Similarities in a fifth-order evolution equation with and with no singular kernel," Chaos, Solitons \& Fractals, vol. 130, Article ID 109467, 2020.

[33] P. Veeresha, D. G. Prakasha, and S. Kumar, "A fractional model for propagation of classical optical solitons by using nonsingular derivative," Mathematical Methods in the Applied Sciences, vol. 2020, 2020.

[34] S. Kumar, "A new analytical modelling for fractional telegraph equation via Laplace transform," Applied Mathematical Modelling, vol. 38, no. 13, pp. 3154-3163, 2013.

[35] S. Kumar, S. Ghosh, B. Samet, and E. F. D. Goufo, "An analysis for heat equations arises in diffusion process using new yangabdel-aty-cattani fractional operator," Mathematical Methods in the Applied Sciences, vol. 2020, 2020.

[36] S. Kumar, R. P. Chauhan, S. Momani, and S. Hadid, "Numerical investigations on COVID-19 model through singular and non-singular fractional operators," Numerical Methods for Partial Differential Equations, vol. 2020, 2020.

[37] S. Kumar, R. Kumar, S. Momani, and S. Hadid, "A study on fractional COVID-19 disease model by using hermite wavelets," Mathematical Methods in the Applied Sciences, vol. 2021, 2021.

[38] S. Kumar, A. Ahmadian, R. Kumar et al., "An efficient numerical method for fractional sir epidemic model of infectious disease by using bernstein wavelets," Mathematics, vol. 8, no. 4 , p. $558,2020$.

[39] H. Liu, K. Zhang, Y. Ye, Y. Wei, and M. Ma, "Dynamic complexity and bifurcation analysis of a host-parasitoid model with allee effect and holling type III functional response," Advances in Difference Equations, vol. 507, 2019.

[40] H. Liu, Y. Ye, Y. Wei, M. Ma, and K. Zhang, "Pattern formation in a reaction-diffusion predator-prey model with weak allee effect and delay," Complexity, vol. 2019, Article ID 6282958, 2019.

[41] Y. Huang, Z. Zhu, and Z. Li, "Modeling the Allee effect and fear effect in predator-prey system incorporating a prey refuge," Advances in Difference Equations, vol. 1, 2020.

[42] Y. Cai, M. Banerjee, M. Banerjee, Y. Kang, and W. Wang, "Spatiotemporal complexity in a predator--prey model with weak allee effects," Mathematical Biosciences and Engineering, vol. 11, no. 6, pp. 1247-1274, 2014.

[43] F. Zhang and Y. Li, "Stability and hopf bifurcation of a delayed-diffusive predator-prey model with hyperbolic mortality and nonlinear prey harvesting," Nonlinear Dynamics, vol. 88, no. 2, 2017. 